\title{
Pathway-resolved photoelectron emission in dissociative ionization of molecules:
} supplementary materials

\author{
X. GonG, ${ }^{1}$ P. HE, ${ }^{2}$ Q. SONG,${ }^{1}$ Q. JI, ${ }^{1}$ K. LIN, ${ }^{1}$ W. ZHANG, ${ }^{1}$ P. LU, ${ }^{1}$ H. PAN, ${ }^{1}$ J. \\ DING, ${ }^{1}$ H. ZENG,${ }^{1,3, *}$ F. HE, ${ }^{2, *}$ AND J. WU ${ }^{1,4^{*}}$
}

${ }^{1}$ State Key Laboratory of Precision Spectroscopy, East China Normal University, Shanghai 200062, China

${ }^{2}$ Key Laboratory of laser Plasmas (Ministry of Education) and Department of Physics and Astronomy, Collaborative innovation center for IFSA (CICIFSA), Shanghai Jiao Tong University, Shanghai 200240, China

${ }^{3}$ Synergetic Innovation Center of Quantum Information and Quantum Physics, University of Science and Technology of China, Anhui 230026, China

${ }^{4}$ Collaborative Innovation Center of Extreme Optics, Shanxi University, Taiyuan, Shanxi 030006, China

*Corresponding author: hpzeng@phy.ecnu.edu.cn, fhe@sjtu.edu.cn,jwu@phy.ecnu.edu.cn

Published 10 June 2016

This document provides supplementary information to "Pathway-resolved photoelectron emission in dissociative ionization of molecules," http://dx.doi.org/10.1364/optica.3.000643.

(C) 2016 Optical Society of America

http://dx.doi.org/10.1364/optica.3.000643.s001

\section{EXPERIMENTAL METHODS}

Our experimental measurements are performed in an ultrahigh vacuum chamber of COLRIMS ("cold target recoil ion momentum spectroscopy"), which allows us to measure the three-dimensional momenta of ejected electrons and ions in coincidence and thus resolve the photoelectron angular distributions (PADs) into various dissociation pathways. The near-infrared femtosecond pulse from a multipass amplifier Ti:sapphire laser system $(25 \mathrm{fs,}$ $790 \mathrm{~nm}, 10 \mathrm{kHz}$ ) is either frequency-doubled using a $150-\mu \mathrm{m}$-thick $\beta$-barium borate ( $\beta$-BBO) crystal to produce ultraviolet (UV) pulse at $395 \mathrm{~nm}$, or frequency-converted to various wavelengths from $400 \mathrm{~nm}$ to $790 \mathrm{~nm}$ using a traveling-wave optical parametric amplifier superfluorescence (TOPAS). As shown in Fig. S1, the zaxis polarized femtosecond laser pulse propagating along the $\mathrm{x}$ axis is focused onto a supersonic gas jet of $\mathrm{H}_{2}$ by a concave silver mirror $(\mathrm{f}=7.5 \mathrm{~cm}$ ) inside the vacuum chamber. The temporal duration of the 395-nm UV pulse in the chamber is measured to be $\sim 60$ fs. The intensity of the laser pulse in the reaction region is determined by tracing the ponderomotive energy $U_{\mathrm{p}}$ and thus the field intensity dependent energy shift of the ATI peaks of the singly ionized $\mathrm{H}_{2}$.

Rather than pre-aligning $\mathrm{H}_{2}$, the molecular orientation, i.e. $\theta_{1+}$ defined with respect to the laser polarization axis, is deduced from the measured ejection direction of $\mathrm{H}^{+}$for the $\mathrm{H}_{2}(1,0)$ channel. We note that the nuclear kinetic energy $E_{\mathrm{N}}$ of the post-ionization dissociation pathway decreases as increasing the wavelength of the laser pulse. The $E_{\mathrm{N}}$ of the post-ionization dissociation pathway overlaps with that of the stretched-bond ionization pathway when the wavelength is longer than the $550 \mathrm{~nm}$, thus these two pathways cannot be experimentally distinguished.

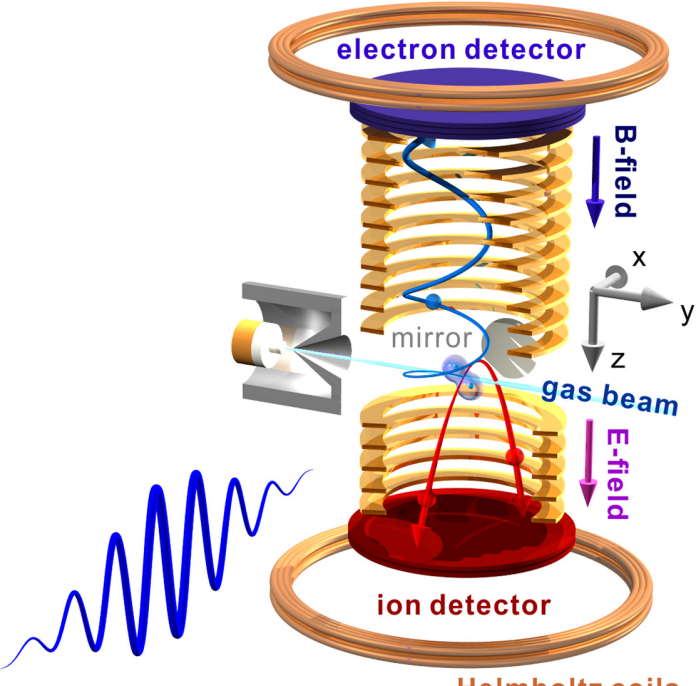

Helmholtz coils

Fig. S1. Schematic diagram of the experimental setup. The laser ionization induced ions and electrons are accelerated by an electric field and detected in coincidence by two detectors at the opposite ends of the spectrometer. To cover a full $4 \pi$ solid angle of the electron detection, a homogenous magnetic field produced from Helmholtz coils is employed. 
As compared to the post-ionization dissociation pathway, the stretched-bond ionization pathway is preferred for long pulse in which the molecule has enough time to elongate in the leading edge of the laser pulse before its ionization at a larger internuclear distance. To test that, a 22-fs UV pulse was generated by frequency doubling a pre-compressed few-cycle pulse from the output of a $\mathrm{Ne}$ gas filled hollow fiber. The UV pulse was alternatively temporally stretched to $112 \mathrm{fs}$ by inserting several pieces of fused silica glasses. As shown in the inset of Fig. 1(a) of the main text, the yield ratio of the stretched-bond ionization pathway is significantly enhanced and suppressed for the 112-fs and 22-fs laser pulses respectively, consisting with the scenario that stretched-bond ionization pathway is produced by releasing an electron at a large internuclear distance.

\section{THEORETICAL METHODS}

We simulated the PADs in the single ionization of $\mathrm{H}_{2}$ at various internuclear distances and orientations with the single active electron approximation by numerically solving the timedependent Schrödinger equation (TDSE) (atomic units are used unless stated otherwise)

$i \frac{\partial}{\partial t} \psi(x, y, z ; t)=$
$\left[-\frac{1}{2} \frac{\partial^{2}}{\partial x^{2}}-\frac{1}{2} \frac{\partial^{2}}{\partial y^{2}}-\frac{1}{2} \frac{\partial^{2}}{\partial z^{2}}+V(R, x, y, z)+z \cdot E(t)\right] \psi(x, y, z ; t)$,

(S1)

where $\mathrm{R}$ is the internuclear distance. The modeled Coulomb potential is written as

$$
\begin{aligned}
& V(R, x, y, z)=-\frac{q}{\sqrt{x^{2}+\left(y-\frac{R}{2} \cos \theta\right)^{2}+\left(z-\frac{R}{2} \sin \theta\right)^{2}+a}}, \\
& -\frac{q}{\sqrt{x^{2}+\left(y+\frac{R}{2} \cos \theta\right)^{2}+\left(z+\frac{R}{2} \sin \theta\right)^{2}+a}}
\end{aligned}
$$

where $\theta$ is the cross angle between the laser polarization direction and the molecular axis, $\mathrm{a}=0.03$ is the soft core parameter, and $\mathrm{q}$ is the effective nuclear charge. We sampled the simulation box by the numerical girds $1500 \times 1500 \times 300$ in $x-y-z$ dimensions with equidistant spacings $\Delta x=\Delta y=\Delta z=0.3$ a.u.. Our simulation box is big enough that no wavepacket hits boundaries during simulations. We used Crank-Nicholson method to propagate the wavepacket. We calculated the single ionization induced PADs when the internuclear distances are $\mathrm{R}=1.4$ a.u. and 6 a.u.. For $\mathrm{R}=6$ a.u. we assumed the screened nucleus has the effective charge $q=0.5$, which gives the ionization potential $I_{\mathrm{p}}=0.23$ a.u.. Thus, the stretched molecule may be ionized by absorbing two or more 395$\mathrm{nm}$ photons. On the other hand, when the internuclear distance is $\mathrm{R}=1.4$ a.u., we set $\mathrm{q}=0.65$ for obtaining the ionization potential $I_{\mathrm{p}}$ $=0.6$ a.u.. The initial states are calculated by imaginary time propagation. This modified Coulomb potential will somehow distort the low energy structure, however, qualitatively describe the PADs for ATI. Though this model cannot precisely produce the electron wave function in the $\mathrm{EF}^{1} \Sigma_{\mathrm{g}}{ }^{+}$state, our test simulations showed the PADs are more sensitive to the molecular orientation instead of the initial wave function. Thus this model can qualitatively explain our experimental measurements.

The laser electric field is $\mathrm{E}=E_{0} \cos (\omega \mathrm{t}) \cos ^{2}\left(\pi \mathrm{t} / \tau_{0}\right),-\tau_{0} / 2<\mathrm{t}<$ $\tau_{0} / 2$ with the pulse duration $\tau_{0}$ being 6 optical cycles for $\omega=0.115$ a.u. (395 nm). The photoelectron momentum is obtained via Fourier transforming the freed wave packets which finally all locate in the area with a distance to the mass center larger than 60 a.u.. The convergence has been tested by propagating the wave function with extra 5 cycles after the laser pulse is finished. 